\title{
Chromosome Analysis of Micaria formicaria (Araneae: Gnaphosidae) from Turkey
}

\author{
Ümit KUMBIÇAK¹, Zübeyde KUMBIÇAK¹, Fahrettin Anıl SIRLIBAŞ²*, Şeyma CIVAN², Hatice POYRAZ² \\ ${ }^{1}$ Nevşehir Hacı Bektaş Veli University, Faculty of Art and Science, Department of Molecular Biology and Genetics, Nevşehir, Turkey \\ ${ }^{2}$ Nevşehir Hacı Bektaş Veli University, Institute of Science, Department of Biology, Nevşehir, Turkey \\ ORCID ID: Ümit KUMBIÇAK: http:/ / orcid.org/0000-0002-1294-3706; Zübeyde KUMBIÇAK: http:/ / orcid.org/0000-0001-5949-1092; Fahrettin \\ Anıl SIRLIBAŞ: http:/ / orcid.org/0000-0002-8071-6441; Şeyma CIVAN: http:/ / orcid.org/0000-0002-3956-3826; Hatice POYRAZ: \\ http://orcid.org/0000-0001-7453-3342
}

\begin{abstract}
In this study, Micaria formicaria (Sundevall, 1831) karyotype properties of the species were analyzed for the first time by using standard giemsa staining method. Gonads derived from the testicles were put through hypotonic application and, fixation and staining processes then, chromosome preparations were prepared. In this study, the number of diploid chromosomes belonging to the species was determined as $2 n=22$. All chromosomes including sex chromosomes are telocentric type; the sexual chromosome system was determined as $\mathrm{X}_{1} \mathrm{X}_{2} \mathrm{O}$. In the meiosis I phases, 10 autosomal bivalent and two heteropicnotic sexual chromosomes with at least one chiasm point were observed. In meiosis II phases, sexual chromosomes showed an isopicnotic feature.
\end{abstract}

Keywords: Spider, karyotypes, cytogenetic, meiosis.

\section{Türkiye'den Micaria formicaria (Araneae: Gnaphosidae) Türünün Kromozom Analizi}

\begin{abstract}
Öz: Bu çalışmada Micaria formicaria (Sundevall, 1831) türünün karyotip özellikleri, standart giemsa boyama yöntemi kullanılarak ilk kez analiz edilmiştir. Testislerden elde edilen gonadlar hipotonik uygulama, fiksasyon ve boyama işlemlerinden geçirilerek kromozom preparatları hazırlanmıştır. Çalışmada türe ait diploid kromozom sayısı $2 \mathrm{n}=22$ olarak belirlenmiştir. Eşey kromozomları da dâhil tüm kromozomlar telosentrik tipte olup eşey kromozom sistemi $\mathrm{X}_{1} \mathrm{X}_{2} 0$ olarak tespit edilmiştir. Mayoz I evrelerinde en az bir kiyazma noktası bulunan 10 otozomal bivalent ve heteropiknotik özellikte iki eşey kromozomu görülmüştür. Mayoz II evrelerinde ise eşey kromozomları izopiknotik özellik göstermiştir.
\end{abstract}

Anahtar kelimeler: Örümcek, karyotip, sitogenetik, mayoz.

\section{Giriş}

Örümcekler, şimdiye kadar tanımı yapılmış 49200 tür ile hayvanlar âlemindeki en büyük takımlardan biri olarak kabul edilmektedir (World Spider Catalog, 2021). Artan çalışmalarla birlikte her gün yeni türler keşfedilmekte ve tanımlanan tür sayısının 170.000 'e kadar ulaşması beklenmektedir (Sebastian \& Mathew, 2009).

Gnaphosidae familyası, 162 cins ve 2566 tür ile örümcek takımı içerisinde bulunan yedinci büyük aile olarak bilinmektedir (World Spider Catalog, 2021). Gnaphosidae familyası üzerine yapılan sitogenetik çalışmaların tanımlanan tür sayısı ile karşılaştırıldı̆̆ında yeterli olmadığı görülmektedir. Günümüzde karyotip özellikleri bilinen sadece 54 gnafozid türü bulunmaktadır (Araujo et al., 2020). Çalışılan bu türlerde diploid kromozom sayısı 2 n=22 ile 30 arasında değişmektedir. Kromozom morfolojisi akrosentrik tipte olup eşey kromozom sistemi ${ }_{0}^{1} \mathrm{X}_{1} \mathrm{X}_{2}$ ve ${ }^{\circ} \mathrm{X}_{1} \mathrm{X}_{1} \mathrm{X}_{2} \mathrm{X}_{2}$ olarak belirlenmiştir (Araujo et al., 2020). Kaydedilen bu özellikler çalışılan türlerin büyük çoğunluğunda görülmektedir. Ancak Drassodes lutescens (L. Koch, 1839) (Kumbiçak et al., 2014) Urozelotes rusticus (L. Koch,1872) (Srivastava \& Shukla, 1986) ve tanımı yapılmamış iki Drassodes (Srivastava \& Shukla, 1986) türünde diploid kromozom sayıs 2 n=21 ve $\mathrm{X} 0$ eşey kromozom sistemi gibi özelliklere nadiren de olsa rastlanmaktadır.

Gnaphosidae familyası ülkemizde 32 cins ve 147 tür ile temsil edilmektedir (Danışman, Kunt, \& Öztürk, 2019). Gnafozidler, Lycosidae ve Salticidae ile ülkemizde sitogenetik olarak en çok çalışılan örümcek ailelerinden biridir. Gnafozid örümcekler üzerine yapılan sitogenetik analizlere ülkemizde yapılan çalışmalarla büyük katkı sağlanmaktadır. Karyotip özellikleri belirlenen 54 türün \%44'ünün (24 tür) veri girişi ülkemizdeki çalışmalar sonucu yapılmıştır (Araujo et al., 2020). Ayrıca ülkemizde Zelotes aeneus (Simon, 1878) türünde kaydedilen 2n=20 diploid kromozom sayısı ve Drassodes lutescens türünde kaydedilen $2 n=21$ ve $X 0$ eşey kromozom sistemi Gnaphosidae familyası türleri arasındaki farklı karyotip özelliklerinin anlaşılması açısından önem arz etmektedir (Taşdemir, Varol, \& Akpınar, 2012; Kumbıçak et al., 2014).

$\mathrm{Bu}$ çalışmayla Gnaphosidae familyasına ait Micaria formicaria (Sundevall, 1831) türünün sitogenetik özelliklerinin analiz edilmesi ile elde edilen verilerin uluslararası sitogenetik veri bankasına girişi yapılarak Gnaphosidae familyası sitogenetik verilerine katkı sağlanması amaçlanmaktadır. 


\section{Materyal ve Metot}

Micaria formicaria türüne ait örneklerin toplandığı arazi çalışması örümceklerin üreme davranışlarının arttığı Mart - Mayıs (2018) aylarında gerçekleşmiştir. Örümcekler doğrudan elle toprak yüzeyinden ve taş altlarından canlı olarak yakalanmıştır. Arazi çalışması; Adana-Pozantı, Mersin-Gülek, Kahramanmaraş-Göksun olmak üzere 3 farklı alanda gerçekleştirilmiştir (Tablo 1). Toplanan örnekler ayrı plastik tüpler içerisinde canlı olarak laboratuvara getirilmiştir. Örneklerin tür teşhisleri pedipalp yapısına ve vücut desenlerinin özelliklerine göre bazı literatürler kullanılarak (Miller, 1971; Heimer \& Nentwig, 1991; Roberts, 1995; Almquist, 2006) Dr. Zübeyde KUMBIÇAK tarafından yapılmıştır. Çalışmada kullanılan örnekler Nevşehir Hacı Bektaş Veli Üniversitesi, Fen Edebiyat Fakültesi, Genetik Araştırma Laboratuvarında muhafaza edilmektedir.

Tablo 1. Micaria formicaria örneklerinin toplandığı lokalitelerin koordinatları.

Table 1. The localities coordinates of Micaria formicaria specimens were collected.

\begin{tabular}{ccc}
\hline Örnek sayısı & Toplama Tarihi & Lokalite Bilgileri \\
\hline $3 \hat{\jmath}$ & 26 Mart 2018 & Adana, Pozantı \\
& & $37^{\circ} 25^{\prime} 35^{\prime \prime} \mathrm{N}, 34^{\circ} 51^{\prime} 42^{\prime \prime} \mathrm{E}$ \\
$5 \hat{\jmath}$ & Mersin, Gülek \\
& 1 Nisan 2018 & $37^{\circ} 15^{\prime} 47^{\prime \prime} \mathrm{N}, 34^{\circ} 45^{\prime} 15^{\prime \prime} \mathrm{E}$ \\
$2 \hat{\sigma}$ & \multirow{2}{*}{ 12 Mayıs 2018 } & Kahramanmaraş, Göksun \\
& & $38^{\circ} 00^{\prime} 32^{\prime \prime} \mathrm{N}, 36^{\circ} 28^{\prime} 57^{\prime \prime} \mathrm{E}$ \\
\hline
\end{tabular}

Bu çalışma, Bedo (1984) metodunda bazı değişiklikler yapılarak gerçekleştirilmiştir. Erkek örümcekler, pedisel bölgesinden ayrılarak öldürülmüş ve opistosoma kısmindan gonadlar elde edilmiştir. Gonadlar, 2-3 ml hipotonik çözelti $(0.075 \mathrm{M} \mathrm{KCl})$ eklenmiş tüplerin içerisinde 40 dakika bekletilmiştir. Süre sonunda 2 defa olmak üzere 2000 rpm'de 5'er dakika süreyle santrifüj yapılmış ve her defasında süpernatant kısım uzaklaştırılmıştır. Fiksasyon aşamasına hazır duruma gelen gonadların bulunduğu tüpe fiksatif eklenerek karıștırılmış ve $2000 \mathrm{rpm}^{\prime}$ de 2 defa olmak üzere 10'ar dakika santrifüj yapılmıştır. Süpernatant kısım atıldıktan sonra elde edilen materyalin üzerine $1 \mathrm{ml}$ fiksatif eklenerek karıştırılmış ve karışımdan bir miktar alınarak lam üzerine bırakılmıştır. Preparatlar havada kurumaya bırakılmış daha sonra en az bir gün olmak üzere buzdolabında $\left(+4^{\circ} \mathrm{C}\right)$ bekletilmiştir. Elde edilen tüm kromozom preparatları faz kontrast mikrobunda incelenerek hücre bölünmesi içeren preparatlar tespit edilmiştir. Bu preparatlar daha sonra fosfat tampon içeren $\% 5^{\prime}$ lik Giemsa boyası $(\mathrm{pH}=6.8)$ ile 50 $\mathrm{dk}$ boyanmıștır. Boyama işlemi sonunda preparatlar sirasılyla musluk suyu ve distile su ile yıkanarak RT'de kurumaya bırakılmıştır. Preparatlar mikroskop incelemesi yapilıncaya kadar buzdolabında $\left(+4^{\circ} \mathrm{C}\right)$ muhafaza edilmiştir.

Hazırlanan preparatlar Olympus CX21 araştırma mikroskobunda 10X büyütmede incelenerek erkek ve dişi bireyler için mitotik metafaz ve mayoz evreleri tespit edilmiştir. Kromozomların ayrıntılı olarak incelenmesi ise 100X büyütmede gerçekleştirilmiştir.

Micaria formicaria türüne ait karyotip yapılması aşamasında en az 10 metafaz evresine ait fotoğraflar, Olympus BX53 araştırma mikroskobu ve DP26 kamera sistemi, CellSens programı (Olympus) ile çekilmiştir. Kromozomların uzunlukları CellSens programı ile ölçülmüş ve kromozomların sentromer konumları Levan et al., (1964)'ye göre belirlenmiştir. Kromozomların çiftler halinde siralanması ise Adobe Photoshop CS6 programı ile gerçekleştirilmiştir.

\section{Bulgular}

\subsection{Karyotip ve Eşey Kromozomu Sistemi}

Micaria formicaria türünün erkek bireylerinin mitotik metafaz evresinde diploid kromozom say $1512 n=22$ olarak tespit edilmiştir (Şekil 1). Eşey kromozom sistemi $X_{1} X_{2} 0$ ve kromozom morfolojisi telosentrik tiptedir. Otozomal kromozom çiftlerinin relatif uzunlukları \%11.98 ile \%6.59 arasında değişmektedir. İkinci büyük kromozom çiftinden başlayarak en küçük çifte doğru kademli bir azalma görülmektedir. Eşey kromozomlarının relatif uzunlukları $\mathrm{X}_{1}$ için \%8.11 ve $\mathrm{X}_{2}$ için \%7.24 olarak belirlenmiştir (Tablo 2).

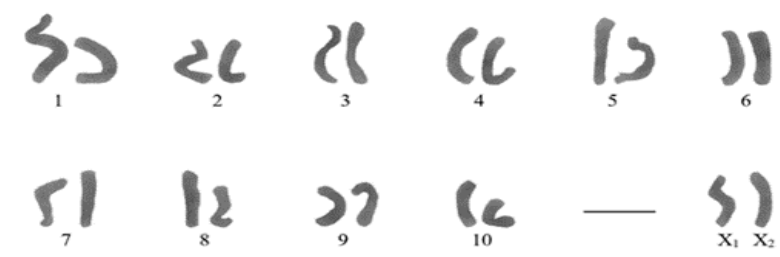

Şekil 1. Micaria formicaria ait karyogram, 10 çift otozomal kromozomlar ve $\mathrm{X}_{1} \mathrm{X}_{2}$ şeklindeki eşey kromozomları (Ölçüm=10 $\mu \mathrm{m})$.

Figure 1. Karyogram of Micaria formicaria, 10 pairs of autosomal chromosomes and $\mathrm{X}_{1} \mathrm{X}_{2}$ shaped sex chromosomes (Measurement $=10 \mu \mathrm{m})$.

Tablo 2. Micaria formicaria türünün erkek bireyine ait karyotipte kromozom uzunlukları (p-kısa kol, q-uzun kol, p+q-toplam uzunluk, q/p-kol oranı), morfolojileri.

Table 2. Chromosome lengths ( $\mathrm{p}$-short arm, $\mathrm{q}$-long arm, $\mathrm{p}+\mathrm{q}$-total length, $\mathrm{q} / \mathrm{p}$-arm ratio) and morphologies in karyotype of male individual of Micaria formicaria species.

\begin{tabular}{|c|c|c|c|c|c|}
\hline $\begin{array}{c}\text { Kromozom } \\
\text { No }\end{array}$ & $p(\mu \mathrm{m})$ & $q(\mu \mathrm{m})$ & $q / p$ & $\begin{array}{l}\text { Oransal } \\
\text { boy }(\%)\end{array}$ & $\begin{array}{l}\text { Kromozom } \\
\text { morfolojisi }\end{array}$ \\
\hline 1 & 0 & $\begin{array}{r}19.19 \\
\pm 3.76\end{array}$ & $\infty$ & 11.98 & Telosentrik \\
\hline 2 & 0 & $\begin{array}{r}14.63 \\
\pm 2.42\end{array}$ & $\infty$ & 9.13 & Telosentrik \\
\hline 3 & 0 & $\begin{array}{r}14.20 \\
\pm 2.57\end{array}$ & $\infty$ & 8.86 & Telosentrik \\
\hline 4 & 0 & $\begin{array}{r}13.92 \\
\pm 2.41\end{array}$ & $\infty$ & 8.69 & Telosentrik \\
\hline 5 & 0 & $\begin{array}{l}13.48 \\
\pm 2.26\end{array}$ & $\infty$ & 8.41 & Telosentrik \\
\hline 6 & 0 & $\begin{array}{r}12.81 \\
\pm 1.83\end{array}$ & $\infty$ & 7.99 & Telosentrik \\
\hline 7 & 0 & $\begin{array}{l}12.48 \\
\pm 1.99\end{array}$ & $\infty$ & 7.79 & Telosentrik \\
\hline 8 & 0 & $\begin{array}{r}11.94 \\
\pm 1.65\end{array}$ & $\infty$ & 7.45 & Telosentrik \\
\hline 9 & 0 & $\begin{array}{r}11.56 \\
\pm 1.59\end{array}$ & $\infty$ & 7.21 & Telosentrik \\
\hline 10 & 0 & $\begin{array}{r}10.56 \\
\pm 1.19\end{array}$ & $\infty$ & 6.59 & Telosentrik \\
\hline $\mathrm{X}_{1}$ & 0 & $\begin{array}{l}13.00 \\
\pm 2.42\end{array}$ & $\infty$ & 8.11 & Telosentrik \\
\hline $\mathrm{X}_{2}$ & 0 & $\begin{array}{r}12.40 \\
\pm 2.42\end{array}$ & $\infty$ & 7.74 & Telosentrik \\
\hline
\end{tabular}

\subsection{Bazı Mitotik ve Mayotik Evrelerinin İncelenmesi}

Mitotik metafaz evresinde yoğunlaşarak tam şeklini alan kromozomlar sayılabilir durumdadır. Bu evrede diploid kromozom sayısı $2 n=22$, kromozom morfolojisi ise eşey 
kromozomları da dâhil telosentrik olarak tespit edilmiştir (Şekil 2a).

Mayotik Profaz I'in alt evresi leptotenden sonra eşey kromozomları vezikül hâlinde belirmeye başlayıp ilerleyen evrelerde kısalıp kalınlaşmaya bağlı olarak sayılabilir duruma gelmektedir. Bu sebeple; zigoten (Şekil $2 b$ ) ve pakiten (Şekil 2c) evrelerinde kısalıp kalınlaşmaya başlayan eşey kromozomları daha koyu boyanarak pozitif heteropiknotik özellik göstermektedir. Ayrıca pakiten evresinde eşey kromozomlarının terminal uçları ile birbirlerine bağlı oldukları görülmektedir (Şekil 2c). Diploten ve diyakinezde en az bir kiyazma noktası bulunan 10 otozomal bivalent ve 2 eşey kromozomu belirlenmiştir (Şekil 2d). Eşey kromozomları pozitif heteropiknotik özellik göstererek çekirdek yüzeyinde konumlanmaktadır. Anafaz I'de koyu boyanmayan eşey kromozomları izopiknotik özellikte olması sebebiyle otozomlardan ayırt edilememektedir. Bu evrede tüm kromozomlar telosentrik morfolojiden ötürü " $\mathrm{V}$ " şeklindedir ve $n=11$ ( 10 otozom $\left.+X_{1} X_{2}\right)$ ve $n=10$ (otozomlar) olmak üzere iki yeni çekirdek kaydedilmiştir (Şekil 2e). Anafaz II evresinde ikisi $n=11$ diğer ikisi n=10 olmak üzere dört yavru çekirdek "I" şeklinde görülmektedir (Şekil 2f).

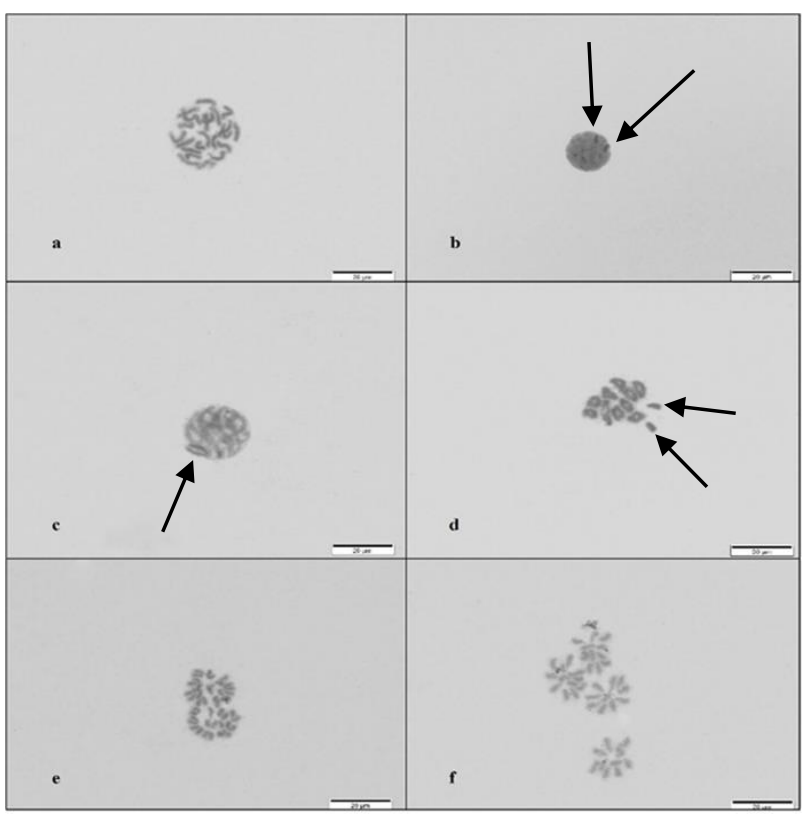

Şekil 2. a. Mitotik metafaz, 2nô: 22, b. Zigoten, c. Pakiten, d. Diploten, 10 otozomal bivalent ve iki eşey kromozomu, e. Anafaz I, f. Anafaz II.

Figure 2. a. Mitotic metaphase, 2n Diplotene, 10 autosomal bivalent and two sex chromosomes, e. Anaphase I, f. Anaphase II.

\section{Tartışma ve Sonuç}

Örümcekler üzerine yapılan sitogenetik çalışmalar yüzyıldan fazla süredir devam etmesine rağmen bu takımla ilgili veriler istenilen düzeyde değildir. Kromozomların elde edilmesindeki zorluklar ve kromozom yapılarının küçük olması gibi olumsuzluklar nedeniyle sadece 868 türün karyotip analizi yapılmıştır (Araujo et al., 2020).

Örümcekler hakkındaki sitogenetik verilerin büyük çoğunluğu Araneomorphae'nin alt gruplarından biri olan entelejin örümceklere dayanmaktadır (Št'áhlavský et al.,
2020). Mevcut örümcek türlerinin yaklaşı $\% 80^{\prime}$ ini içeren entelejinler, düşük diploid sayı $(2 \mathrm{n}=10-49)$ (Kořínková \& Král, 2013), kiyazmatik mayoz (Kumbiçak, 2010) ve akrosentrik kromozom morfolojisinin baskın olduğu bir karyotiple nitelendirilmektedir (Araujo et al., 2020). Gnaphosidae familyası $2 n=22-30$ arasında değişen kromozom sayısı ve genellikle akrosentrik tipteki kromozom morfolojisi ile entelejin örümcekler için karakteristik olan karyolojik özellikleri göstermektedir. Gnaphosidae familyasının eşey kromozom sistemi ${ }_{0}^{3} \mathrm{X}_{1} \mathrm{X}_{2}$ ve ${ }_{+} \mathrm{X}_{1} \mathrm{X}_{1} \mathrm{X}_{2} \mathrm{X}_{2}$ şeklinde tespit edilmiş olup bu sistem $\mathrm{X}_{1} \mathrm{X}_{2} 0$ eşey kromozom sistemi olarak bilinmektedir (Araujo et al., 2020).

Gnaphosidae familyasından sitogenetik analizler sonucu elde edilen veriler homojenlik göstermektedir. Ancak Drassodes lutescens (L. Koch, 1839) (Kumbiçak et al., 2014), Urozelotes rusticus (L. Koch,1872) (Srivastava \& Shukla, 1986) ve tanımı yapılmamış iki Drassodes (Srivastava \& Shukla, 1986) türünde diploid kromozom sayıs $2 n=21$ ve $X 0$ eşey kromozom sistemi gibi familya genelinden farklı özelliklerde kaydedilmiştir. Bu farklılı̆̆ı nedenleri arasında; $X_{1} X_{2} 0$ eşey kromozom sistemindeki $\mathrm{X}$ kromozomlarından birinin aşamalı olarak ortadan kalkması veya $X$ kromozomları arasında distal ya da proksimal füzyondan önce meydana gelen karşllıklı translokasyon gösterilmektedir (Maddison \& LeducRobert, 2013).

Bu çalışmada Türkiye'den toplanan $M$. formicaria türünün karyotip özellikleri ilk kez analiz edilmiştir. Türün diploid kromozom sayısı 2 n=22, eşey kromozom sistemi $\mathrm{X}_{1} \mathrm{X}_{2} \mathrm{O}$ ve kromozom morfolojisi telosentrik olarak bulunmuştur. Mayotik Profaz I'den itibaren vezikül halinde belirmeye başlayan eşey kromozomları pozitif heteropiknotik özellik göstermelerinden dolayı otozomlardan ayırt edilebilmektedir. Sonuç olarak elde edilen bu karyolojik veriler Gnaphosidae familyasına ait önceki verilerle uyumluluk göstermektedir.

Etik kurul onayı: Bu çalışma için etik kurul onayı alınmasına gerek yoktur.

Çıkar çatışması: Yazarlar, çıkar çatışması olmadığını beyan etmiştir.

\section{Kaynaklar}

Almquist, S. (2006). Swedish Araneae, part: 2 families Dictynidae to Salticidae. Insect Systematics \& Evolution, Supplement 63, 285-601.

Araujo, D., Schneider, M.C., Paula-Neto, E., \& Cella, M.D. (2020). The spider cytogenetic database. Retrieved from http://www.arthropodacytogenetics.bio.br/spiderdatabase/

Bedo, D.G. (1984). Karyotypic and chromosome banding studies of the potato tuber moth, Phthorimaea operculella 43 (Zeller) (Lepidoptera, Gelechiidae). Canadian Journal of Genetics and Cytology, 26(2), 41-145. https://doi.org/10.1139/g84-024

Danışman, T., Kunt, K.B., \& Özkütük, R.S. (2019). The Checklist of the Spiders of Turkey (Araneae; Arachnida). Retrieved from http://www.spidersofturkey.info/index.htm

Heimer, S., \& Nentwig, W. (1991). Spinnen Mitteleuropas: Ein Bestimmungsbuch. Berlin: Paul Parey pp. 543.

Kořínková, T., \& Král, J. (2013). Karyotypes, sex chromosomes, and meiotic division in spiders. In: Nentwig W. (ed.), Spider Ecophysiology. Springer-Verlag, Berlin, 151-159

Kumbiçak , Z., Ergene, S., Kumbiçak, Ü., \& Ekiz, E. (2014). A chromosomal analysis of five spider species (Araneae: Gnaphosidae, Miturgidae and Philodromidae) from Turkey. Caryologia, 67(2), 155-159. https://doi.org/10.1080/00087114.2014.931637 
Kumbıçak, Z. (2010). Türkiye'de Bazı Örümceklerde Karyotip ve Eşey Kromozomlarının Belirlenmesi Üzerine Araştırmalar (Doktora Tezi). Gaziantep Üniversitesi Fen Bilimleri Enstitüsü Biyoloji, Retrieved from https://tez.yok.gov.tr/UlusalTezMerkezi/giris.jsp

Levan, A., Fredga, K., \& Sandberg, A.A. (1964). Nomenclature of centromeric position on chromosomes. Hereditas, 52(2), 201-220. https://doi.org/10.1111/j.1601-5223.1964.tb01953.x

Maddison, W., \& Leduc-Robert, G. (2013). Multiple origins of sex chromosome fusions correlated with chiasma localization in Habronattus jumping spiders (Araneae: Salticidae). Evolution, 67(8), 2258-2272. https://doi.org/10.1111/evo.12109

Miller, F. (1971). Pavouci-Araneida. Klič zvířeny ČSSR, 4, 51-306.

Roberts, M.J. (1995). Collins Field Guide to the Spiders of Britain and Northern Europe. London: HarperCollins pp. 383.

Sebastian , P., \& Mathew, M. (2009). Introduction to Spiders. In: Sebastian P. \& Peter K. (ed) Spiders of India. Universities Press (India) Private Limited, Hyderabad, 1-39.

Srivastava, M., \& Shukla, S. (1986). Chromosome number and sexdetermining mechanism in fortyseven species of Indian spiders. Chromosome Information Service, 41, 23-26.

Štáhlavský, F., Forman, M., Just, P., Denič, F., Haddad, C.R., \& Optova, V. (2020). Cytogenetics of entelegyne spiders (Arachnida, Araneae) from southern Africa. CompCytogen, 14(1), 107-138. https://doi.org/10.3897/CompCytogen.v14i1.48667

Taşdemir, B., Varol, İ., \& Akpınar, A. (2012). Cytotaxonomical studies on six species of spiders (Arachnida: Araneae) from Turkey. Türkiye Entomoloji Bülteni, 2(2), 55-59.

World Spider Catalog. (2021). World Spider Catalog. Version 22.0. Natural History Museum Bern. Retrieved from https://wsc.nmbe.ch/ https://doi.org/10.24436/2 